\title{
Implantación de condrocitos autólogos versus microfracturas para el tratamiento de lesiones del cartílago en la rodilla
}

\author{
Ortega-Orozco R, * Olague-Franco JK, ${ }^{* *}$ Miranda-Ramírez E*** \\ Medyarthros, Medicina Deportiva y Artroscópica
}

RESUMEN. Objetivo: Comparar la eficacia clínica y la seguridad de la terapia de microfracturas (MF) versus implantación de condrocitos autólogos en membrana (MACI) en el manejo de lesiones condrales de rodilla $\geq 3 \mathrm{~cm}^{2}$ y el seguimiento a 12 meses postratamiento. Material y métodos: Se realizó un estudio de cohorte retrospectiva, de Enero de 2016 a Diciembre de 2017. Se incluyeron pacientes con una 0 varias lesiones condrales en rodilla $\geq 3$ $\mathbf{c m}^{2}$ para comparar la terapia MF versus MACI para la reparación de lesión condral. Se realizaron valoraciones clínicas y funcionales previas al tratamiento quirúrgico y 12 meses posteriores, con medición de los arcos de movimiento, escala EVA, Oxford e índice de Lequesne. Resultados: Se incluyeron 12 pacientes en MF y 12 pacientes en MACI. La lesión más frecuente se localizó en la patela en ocho pacientes $(67 \%)$. Se demostró incremento en los arcos de movimiento, así como mejoría en la comparación entre el nivel basal y en el seguimiento a 12 meses: en EVA, MF mostró $48.4 \%$ y MACI $57.5 \%$ (p $\leq \mathbf{0 . 0 5})$; escala de Oxford: MF 32.65\% y MACI 51.04\% ( $\leq 0.05)$; índice de Lequesne: MF 40.12\% y MACI $50 \%$. Se presentaron dos casos de derrame articular en MACI, que se resolvieron
ABSTRACT. Objective: To compare the clinical efficacy and safety of microfracture therapy (MF) versus implantation of autologous chondrocytes (MACI) in the management of chondral lesions of the knee $\geq 3 \mathrm{~cm}^{2}$ and follow up to 12 months post treatment. Material and methods: A retrospective cohort study was conducted from January 2016 to December 2017. Patients with one or more chondral lesions in $\mathrm{knee} \geq \mathbf{3} \mathbf{c m}^{2}$ were included to compare MF versus MACI therapy for the repair of chondral lesion. Clinical and functional evaluations were carried out prior to the surgical treatment and 12 months later, with measurement of the range of motion, EVA, Oxford scale and Lequesne index. Results: Twelve patients were included in MF and 12 patients in MACI. The most frequent lesion was located in the Patella in eight patients $(67 \%)$. It showed an increase in the arcs of motion, as well as improvement in the comparison between baseline and follow-up at 12 months: in EVA, MF demonstrated $48.4 \%$ and MACI $57.5 \%$ (p $\leq 0.05)$; Oxford scale: MF $32.65 \%$ and MACI $51.04 \%(p \leq 0.05)$; index of Lequesne: MF 40.12\% and MACI $50 \%$. Two cases of joint effusion were presented in MACI, which were resolved with the

Nivel de evidencia: IV

\footnotetext{
* Especialista en Traumatología y Ortopedia, Subespecialista en Cirugía Artroscópica y Medicina del Deporte. Director de Medyarthros, Medicina Deportiva y Artroscópica; Jefe del Servicio de Artroscopía y Medicina del Deporte, Hospital Civil de Guadalajara.

** Especialista en Traumatología y Ortopedia, Subespecialista en Cirugía de Hombro y Codo. Especialista de Medyarthros, Medicina Deportiva y Artroscópica.

*** Especialista en Traumatología y Ortopedia, Especialista en Módulo de Rodilla en Medyarthros, Medicina Deportiva.
}

Guadalajara, Jalisco. México.

Dirección para correspondencia:

Dra. Julieth Kristel Olague-Franco

Especialista en Traumatología y Ortopedia, Subespecialista en Cirugía de Hombro y Codo. Médico de Medyarthros,

Medicina Deportiva y Artroscópica.

Guadalajara, Jalisco, México.

E-mail: dbgtz47@gmail.com. ORCID: Orcid.org/0000-0002-4866-3642

Este artículo puede ser consultado en versión completa en http://www.medigraphic.com/actaortopedica 
con la realización de artrotomías. Conclusión: En este estudio se demostró mejoría significativa en MACI con alivio del dolor, funcionalidad y arcos de movimiento en comparación con el tratamiento de MF en lesiones $\geq 3 \mathrm{~cm}^{2}$ del cartílago articular de rodilla después de un año de seguimiento.

Palabras clave: Implantación, condrocitos, microfracturas, lesiones condrales.

\section{Introducción}

La incidencia de las lesiones del cartílago en la rodilla no es conocida; por otro lado, la prevalencia en la población general se reporta en $12 \% .{ }^{1}$ El impacto clínico y la indicación de intervención quirúrgica varían según las características de la(s) lesión(es) y no únicamente por los síntomas. Los pacientes se presentan con dolor, edema y bloqueo de la articulación con pérdida significativa de la función y disminución de la calidad de vida; existe predisposición del cartílago articular lesionado a desarrollar osteoartritis con discapacidades asociadas e impacto socioeconómico. ${ }^{2,3,4}$ Existen varias modalidades terapéuticas con el objetivo de reducir el dolor y restaurar la función; estos tratamientos pueden ser médicos como la terapia farmacológica o fisiátrica y técnicas quirúrgicas que se dividen en reemplazo, estimulación ósea y técnicas de regeneración. El reemplazo se logra con injertos autólogos osteocondrales como la mosaicoplastía; sin embargo, esta técnica está limitada por la toma y el potencial daño en el sitio de obtención del injerto. ${ }^{5}$

Por otro lado, las técnicas de estimulación ósea (microfracturas) tienen como objetivo generar una respuesta tisular al exponer la médula ósea subcondral y producir un coágulo de sangre que permita la migración de células madre mesenquimales para lograr la reparación del cartílago.

La microfractura (MF) se recomienda como tratamiento primario para lesiones condrales focales; estimula la reparación condral y forma tejido fibrocartilaginoso que es mecánicamente inferior al cartílago articular hialino de origen. ${ }^{6,7}$ Diversos estudios reportan alivio del dolor y mejoría funcional con las microfracturas, principalmente se reconoce su efectividad en pacientes con lesiones recientes (síntomas $<12$ meses de evolución), lesiones focales $\left(<2.5 \mathrm{~cm}^{2}\right)$, pacientes jóvenes $(<30$ años) y sin obesidad (IMC $<30 \mathrm{~kg}$ ) $\left.\mathrm{m}^{2}\right){ }^{7,8,9}$ Por otro lado, se ha utilizado el implante de condrocitos autólogos que evolucionó de la primera a la tercera generación; esta técnica regenerativa estimula la producción de tejido con propiedades estructurales y biomecánicas similares al cartílago hialino de origen. La primera generación fue un parche de periostio autólogo que mantenía los condrocitos in situ; los resultados fueron alivio del dolor, pero se presentaron complicaciones como adherencias intraarticulares, hipertrofia perióstica y deslaminación del defecto. ${ }^{10,11}$ En la segunda generación se utilizó una membrana realization of arthrotomies. Conclusion: In this study, significant improvement was demonstrated in MACI with pain relief, functionality, and range of motion compared to the treatment of MF in lesions $\geq 3 \mathrm{~cm}^{2}$ of the articular cartilage of the knee after one year of follow-up.

Key words: Implantation, chondrocyte, microfracture, chondral injuries.

de colágeno reabsorbible, pero de características frágiles y recientemente se encuentra disponible la tercera generación con la implantación de condrocitos autólogos en membrana (MACI; Genzyme Biosurgery, Cambridge, MA, EUA) que reemplaza el parche perióstico por una membrana de colágeno tipo I/III reabsorbible, ${ }^{12}$ sus características fenotípicas ofrecen mayor resistencia a roturas y durabilidad que permite que el implante sea fácilmente manejado y recortado: otros beneficios adicionales son la menor duración del procedimiento, incisiones más pequeñas, cultivo de células más consistente y menor hipertrofia perióstica. ${ }^{13,14}$

Dos estudios compararon las MF versus $\mathrm{MACI}^{15,16}$ y concluyeron que las MF deben preferirse sobre MACI, mientras que Saris y cols. ${ }^{17}$ proponen que MACI es superior a las MF. El contraste en los resultados de los estudios reportados probablemente se relaciona con diferencias metodológicas y en el diseño de los estudios, por lo que no existe una recomendación estándar ampliamente aceptada. El objetivo de este estudio fue comparar la eficacia clínica y la seguridad de la terapia MACI versus microfracturas (MF) en el manejo de lesiones condrales de rodilla $\geq 3 \mathrm{~cm}^{2}$ y el seguimiento a 12 meses postratamiento.

\section{Material y métodos}

El diseño del estudio fue de cohorte retrospectiva; en el período de Enero de 2016 a Diciembre de 2017 fueron incluidos pacientes con tratamiento de MF (en lo sucesivo se describe como grupo MF) o tratamiento con MACI (grupo MACI) por lesiones focales $\geq 3 \mathrm{~cm}^{2}$, grado III o IV del cartílago (índice de Outerbridge) ${ }^{15}$ con localización en la patela, cóndilo lateral femoral y/o la tróclea y al menos un año de evolución, posterior al tratamiento quirúrgico. Los pacientes no fueron asignados aleatoriamente a los grupos de estudio y la elección de ser tratados por MF o MACI fue determinada por los pacientes una vez que fueron informados de las características de cada procedimiento. En todos los pacientes incluidos se demostró estabilidad de la rodilla con ligamentos íntegros, así como menisco medial y lateral en su forma total o parcial $(\geq 50 \%)$ con reparación y/o resección parcial previa, aun cuando estos procedimientos se realizaron durante la reparación del cartílago. Los criterios de no inclusión fueron enfermedades inflamatorias o procesos sépticos articulares. El protocolo fue aprobado por un comité institucional de ética en investigación. 
Se elaboró un formato de registro con las siguientes variables: edad, sexo, peso, índice de masa corporal, localización de la lesión condral, manifestaciones clínicas que incluyeron las características del dolor, edema, bloqueo de la articulación con pérdida de la función y arcos de movimiento de la rodilla. Se utilizó la escala visual análoga (EVA) para estimar el dolor antes y después de la intervención siendo de 0 a 3 leve, de 4 a 6 moderado y de 7 a 10 severo. La escala de Oxford clasifica los resultados con función mala, regular, buena y excelente: el puntaje de 0 es un resultado malo y 48 es excelente; y el índice de Lequesne se utilizó para evaluar el dolor y la función antes y después de la intervención: con un puntaje de 0 se clasificó el resultado como excelente y mayor de 18 como malo. A 12 meses posteriores al tratamiento quirúrgico los pacientes fueron interrogados para conocer su nivel de satisfacción con el tratamiento.

\section{Técnica quirúrgica}

Todos los pacientes incluidos en el estudio fueron intervenidos en la misma clínica y por el mismo cirujano; se

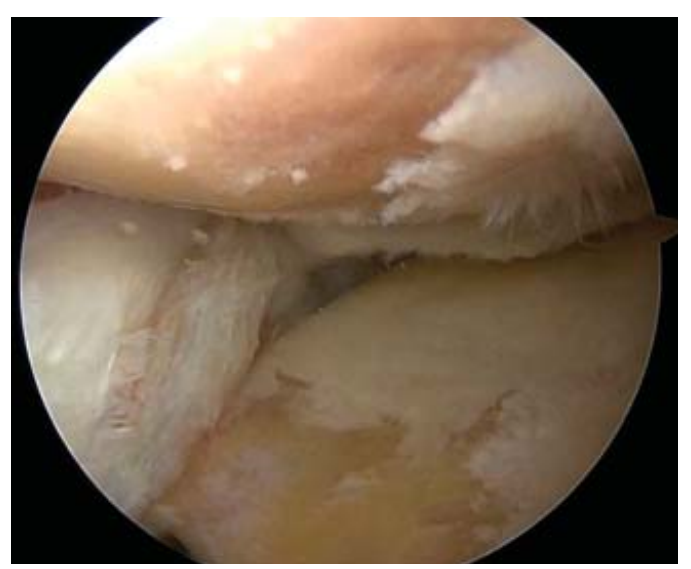

Figura 1: Grupo MF, paciente en decúbito supino al que se le realiza una artroscopía donde se localiza lesión en cóndilo femoral lateral $>3 \mathrm{~cm}^{2}$.

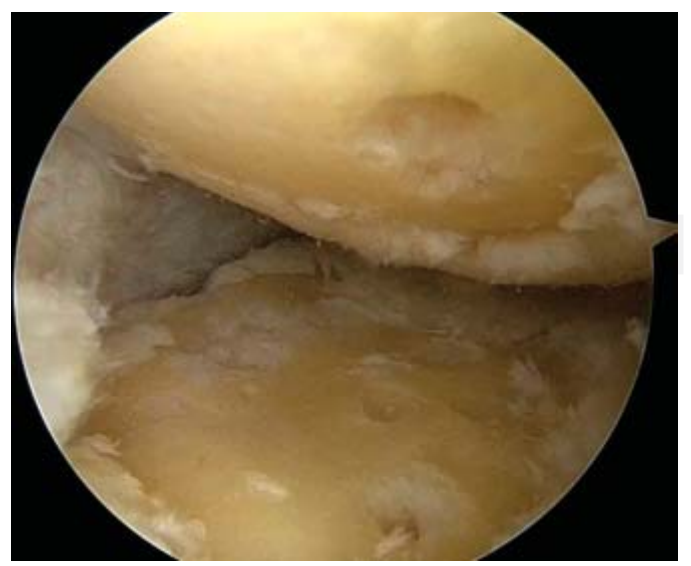

Figura 2: Se realizan microperforaciones con un diámetro de 3-4 mm en la zona de la lesión. sometieron a una primera cirugía artroscópica para evaluar el cumplimiento de los criterios artroscópicos de la lesión. El grupo MF fue sometido a desbridamiento del cartílago y perforaciones múltiples con un punzón agudo en el hueso subcondral dentro de la lesión: concéntricas con 3-4 mm de separación y $4 \mathrm{~mm}$ de profundidad ${ }^{16}$ (Figuras 1 y 2 ).

El grupo MACI fue sometido a biopsia de cartílago; el tejido fue enviado a un banco de huesos para su cultivo durante tres a cuatro semanas y se obtuvo una membrana de colágena de aproximadamente $28 \mathrm{~cm}^{2}\left(7 \times 4 \mathrm{~cm}^{2}\right)$ para cada paciente. El procedimiento de implantación del injerto MACI se realizó a través de miniartrotomía aproximadamente cuatro semanas posteriores a la artroscopía basal. Las lesiones fueron desbridadas hasta el borde de cartílago sano, estable y sin afectar el hueso subcondral; la forma y el tamaño de cada una de las lesiones se midieron para crear una plantilla: enseguida, el injerto MACI fue recortado al tamaño y forma correctos de acuerdo con la lesión del cartílago y se colocó en la base desbridada del defecto con la zona de las células en contacto con el hueso subcondral. El implante se aseguró en el sitio con una capa fina de fibrina como sellador en la base y los bordes del defecto; para concluir el procedimiento se realizó flexión y extensión de la rodilla para verificar la estabilidad del injerto (Figuras 3 y 4 ).

\section{Rehabilitación}

El programa se llevó a cabo con base en la evaluación del fisioterapeuta; se individualizó la progresión de cada paciente de acuerdo con el tamaño y ubicación de la lesión, síntomas preoperatorios, condición física, dedicación del paciente al programa de rehabilitación y la evolución esperada de acuerdo con el procedimiento realizado.

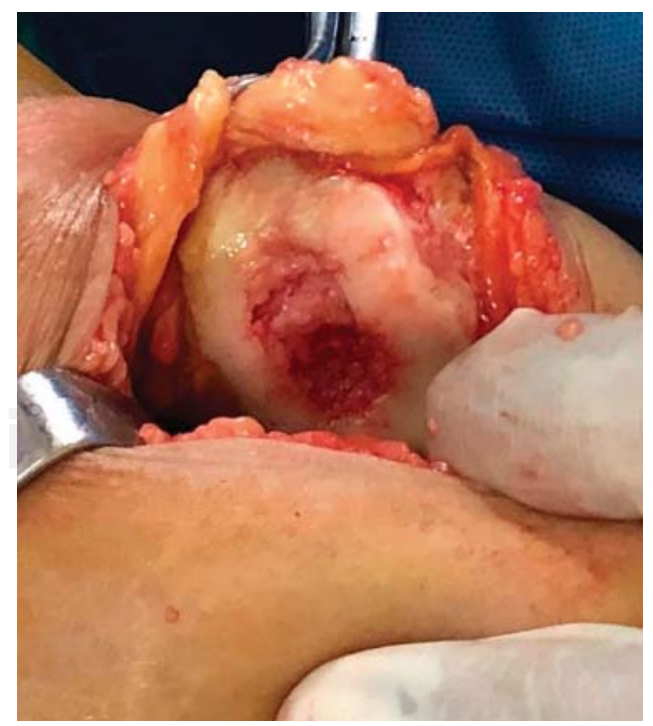

Figura 3: Grupo MACI, paciente en decúbito supino al que se le realiza una artrotomía para rotuliana de $4 \mathrm{~cm}$ aproximadamente, se localiza lesión en patela $>3 \mathrm{~cm}^{2}$. 


\section{Análisis estadístico}

Los resultados se presentan en frecuencias, porcentajes y rangos analizados con Excel (Microsoft Seattle, WA. EUA). El valor de $\mathrm{p} \leq 0.05$ se definió como significativo.

\section{Resultados}

Se incluyeron 24 pacientes; 12 en el grupo MF y 12 en el grupo MACI. La edad promedio fue comparable (41.58 versus 41.83 , respectivamente); predominaron las mujeres y el sobrepeso en ambos grupos, aunque en el grupo MF tres pacientes fueron obesos grado I (Tabla 1). La lesión más frecuente se localizó en la patela en ocho pacientes (67\%) de cada grupo; se compararon los valores basales y seis meses posteriores con ambos procedimientos al concluir el manejo fisiátrico y su mejoría con incremento en los arcos de movimiento en la flexión y extensión.

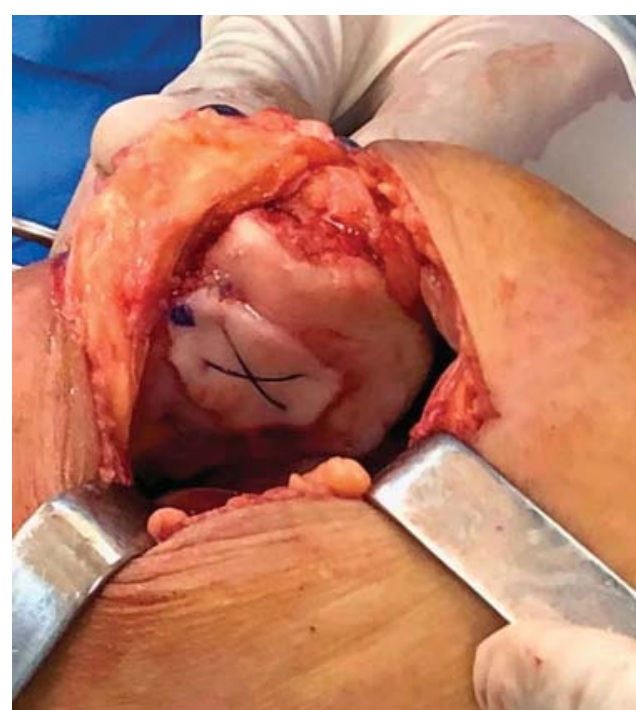

Figura 4: Se realiza marcaje y recorte de plantilla MACI a tamaño de la lesión y se fija con suturas absorbibles.

\section{Valoración de las escalas funcionales y del dolor}

La escala de EVA fue evaluada previa a la cirugía; el promedio en el grupo MF fue de 7.5 (rango 5-9), mientras que en el grupo MACI fue de 7.83 (rango 5-10), esta diferencia no fue significativa (Figuras 5 y 6); en el seguimiento, a 12 meses del tratamiento quirúrgico se obtuvo mejoría significativa en ambos grupos $(\mathrm{p}<0.001)$ con EVA de 2.66 (rango 0-7) en el grupo MF y 2.33 (rango 0-5) en el grupo MACI. La magnitud proporcional de la mejoría fue mayor en el grupo MACI, comparada con el grupo MF (57.5 versus $48.4 \%$, respectivamente) $(\mathrm{p}<0.05)$.

La evaluación funcional y del dolor con la escala Oxford mostró mejoría significativa, comparando los parámetros basales previos al procedimiento quirúrgico con el seguimiento a 12 meses; en el grupo MF se obtuvo 22.16 (rango 7-34) y 37.83 (rango 12-34), respectivamente (Figura 7). En el grupo MACI se obtuvo un promedio basal de 22.25 (rango 28-47) y 44.75 (rango 36-48) a 12 meses de seguimiento ( $\mathrm{p} \leq 0.001)$ (Figura 8). La mejoría fue mayor en el grupo MACI, comparado con el grupo MF (51.04 versus $32.65 \%$, respectivamente $)(\mathrm{p}<0.05)$.

Con el índice de Lequesne se demostró mejoría para el grupo MF con $40.12 \%$, comparado con $50 \%$ para el grupo MACI ( $<20.05$ ); con una evaluación basal de 13.58 (rango 8-16) para MF y 13.41 (rango 8-17) para MACI con un resultado 12 meses después de 4.75 (rango 1-14) y 2.41 (rango 0-9), respectivamente $(\mathrm{p} \leq 0.001)$ (Figuras 9 y 10).

Se destaca que los pacientes del grupo MACI en las evaluaciones sintomáticas y funcionales iniciaron con un rango de regular y malo y después de un año del tratamiento quirúrgico tuvieron un resultado de bueno y excelente; asimismo, los pacientes refirieron estar satisfechos con su tratamiento.

\section{Seguridad}

Durante el estudio se reportaron dos casos de derrame articular en el grupo MACI que se resolvieron con artroto-

\begin{tabular}{lcc}
\hline \multicolumn{2}{c}{ Tabla 1: Características de los pacientes en los dos grupos de estudio. } \\
& Grupo microfracturas $n=12$ & Grupo MACI $\mathrm{n}=12$ \\
& & $41.83($ rango $18-58)$ \\
Edad & $41.58($ rango $26-57)$ & $3(25 \%) / 9(75 \%)$ \\
Sexo (hombres/mujeres) & $5(41.66 \%) / 7(58.33 \%)$ & $24.92 \%($ rango $20.94-28.73)$ \\
IMC & $26.94 \%($ rango 21.5-34.9) & 8 \\
Localización de las lesiones de rodilla & 8 & 3 \\
Patela & 1 & 4 \\
Tróclea & 3 & $100.41^{\circ}$ \\
Cóndilo femoral lateral & $113.33^{\circ}$ & $133.33^{\circ}$ \\
Comparación de los arcos de movimiento entre los previos a la intervención quirúrgico y seis meses posteriores & $1.25^{\circ}$ \\
Flexión basal & $127.41^{\circ}$ & $1.25^{\circ}$ \\
Flexión seis meses posteriores a la cirugía & $0.83^{\circ}$ & $0.33^{\circ}$
\end{tabular}


mías realizadas en las dos semanas posteriores a la cirugía; la evolución fue satisfactoria.

\section{Discusión}

En nuestro estudio se demostró mejoría significativa en los pacientes tratados con MACI con alivio del dolor, funcionalidad y arcos de movimiento comparado con MF en las lesiones $\geq 3 \mathrm{~cm}^{2}$ del cartílago articular de rodilla después de

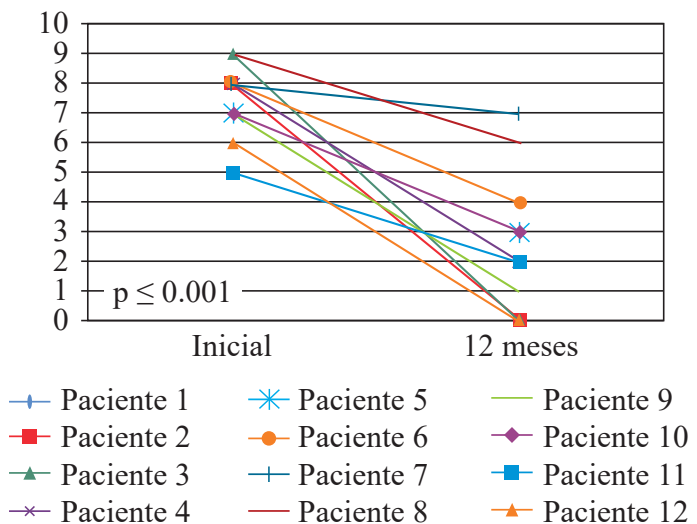

Figura 5: Grupo de microfracturas: escala EVA.

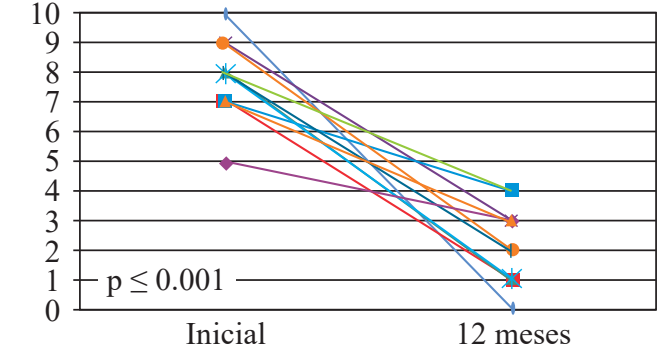

$\begin{array}{lll}\rightarrow \text { Paciente } 1 & * \text { Paciente } 5 & - \text { Paciente } 9 \\ \leftarrow \text { - Paciente } 2 & - \text { - Paciente } 6 & - \text { Paciente } 10 \\ \leftarrow \text { Paciente } 3 & \leftarrow \text { Paciente } 7 & - \text { Paciente } 11 \\ \leftarrow \text { Paciente } 4 & - \text { Paciente } 8 & - \text { Paciente } 12\end{array}$

Figura 6: Grupo de MACI: escala EVA.

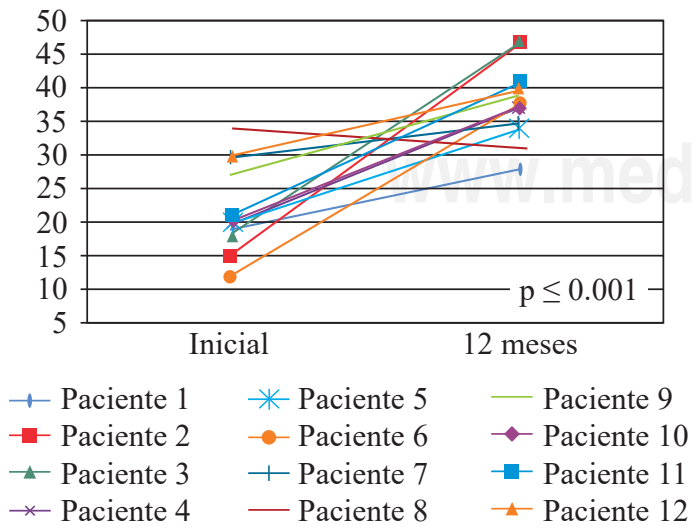

Figura 7: Grupo de microfracturas: escala de Oxford. un año de seguimiento. Nuestra hipótesis es que la capacidad de MACI para generar cartílago hialino, reportado previamente, se relaciona con las diferencias en los resultados observados en nuestros pacientes, en contraste con el mecanismo de las microfracturas que genera fibrocartílago. ${ }^{17,18}$

El tratamiento con MACI está indicado para el manejo de las lesiones $\geq 3 \mathrm{~cm}^{2}$ del cartílago articular, aunque no hay consenso para establecerlo como procedimiento estándar. Existen varias publicaciones que han demostrado resultados clínicos y funcionales superiores al tratamiento con micro-



Figura 8: Grupo de MACI: escala de Oxford.



Figura 9: Grupo de microfracturas: índice de Lequesne.

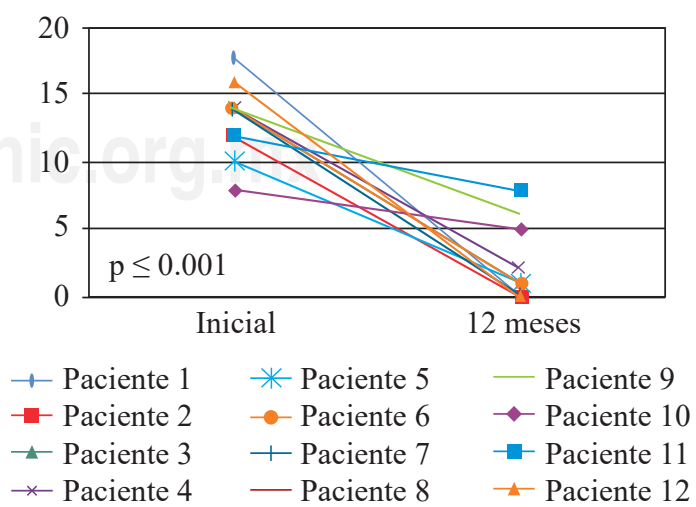

Figura 10: Grupo de MACI: índice de Lequesne. 
fracturas. ${ }^{17,18,19} \mathrm{E}$ l tratamiento con MF aún es considerado por algunos como terapia de primera línea para la reparación del cartílago, es fácil de realizar, de alta disponibilidad y es ampliamente utilizado, lo que refleja una experiencia pragmática del «mundo real» en el tratamiento de las lesiones condrales; ${ }^{16}$ sin embargo, la respuesta terapéutica a MF es altamente variable, condicionada por la presencia de hueso sano para el suministro de células mesenquimales provenientes de las perforaciones del hueso subcondral; puede ocurrir inconsistencia en la formación de fibrocartílago, por lo que es particularmente útil en lesiones pequeñas $\left(<3 \mathrm{~cm}^{2}\right) .{ }^{17}$ En el presente estudio los pacientes con lesiones $\geq 3 \mathrm{~cm}^{2}$ del cartílago articular de rodilla tratados con MACI mostraron mejoría en la escala de EVA comparable con MF, aunque fue mayor la magnitud proporcional en la disminución del dolor para el grupo MACI comparado con MF (57.5 versus 48.4\%, respectivamente). Las ventajas de esta escala son: es sencilla, fácil de aplicar y ampliamente utilizada para evaluar el dolor en todas las áreas clínicas. En un estudio de Guillén y cols. ${ }^{18}$ se informó que $35(70 \%)$ de 50 pacientes tuvieron un puntaje $<3$ (dolor leve) después de dos años del tratamiento. En el estudio que nos ocupa la evaluación funcional con la escala de Oxford mostró resultados excelentes con MACI (pretratamiento 22.5 versus 12 meses postratamiento 44.75) y buenos con MF (pretratamiento 22.16 versus 12 meses postratamiento 37.83) ( $\mathrm{p}<0.001)$; la mejoría fue mayor en el grupo MACI en comparación con el grupo MF (51.04 versus 32.65\%, respectivamente). Las puntuaciones con el índice de Lequesne también mejoraron significativamente con MACI en comparación con $\mathrm{MF}$; fue relevante la evolución funcional con un promedio de $40.12 \%$ para MF y $50 \%$ para el grupo MACI $(\mathrm{p}<0.05)$. Además, MACI también mostró buenos resultados en la recuperación de los arcos de movimiento, aunque no fue significativamente diferente con MF.

Nuestros resultados clínicos y funcionales con MACI versus MF son consistentes con un estudio aleatorizado en pacientes con defectos condrales sintomáticos en cóndilo femoral y rótula $(n=60)$; en el seguimiento a dos años se demostró que MACI fue significativamente más efectivo que $\mathrm{MF} ;{ }^{19}$ en el grupo MACI se observó mejoría clínica con la evaluación de la escala Lysholm con un nivel basal promedio de 52 que se incrementó a 95 al cabo de 12 meses y la mejoría se mantuvo por dos años: en contraste, en el grupo MF la mejoría fue menor, el promedio basal fue de 55 que se incrementó a 81 al cabo de 12 meses, aunque esta mejoría no se mantuvo a los dos años con un resultado promedio que disminuyó a 69. Los resultados clínicos y funcionales con MACI en nuestro estudio son comparables con los reportados en una cohorte prospectiva de Marlovits y cols., ${ }^{20}$ quienes informaron mejoría clínica y radiológica significativa en 21 pacientes con diagnóstico de lesión condral traumática, sintomática y con seguimiento de cinco años posteriores al tratamiento con MACI. Asimismo, Moseley y cols. ${ }^{21}$ reportaron en un estudio de cohorte con 72 casos mejoría clínica significativa $(\mathrm{p}<0.01) ; 54(77 \%)$ pacientes refirieron estar satisfechos con su tratamiento en un seguimiento de cinco años. El tratamiento con MACI es una opción efectiva para el manejo de lesiones $\geq 3 \mathrm{~cm}^{2}$ del cartílago articular; es una técnica reproducible, segura y con ventajas por el menor tiempo quirúrgico, la simplicidad quirúrgica y es mínimamente invasiva.

Se ha descrito un buen perfil de seguridad para el tratamiento de lesiones condrales con MACI. ${ }^{19,20,21}$ En un reporte se observó presencia de edema postoperatorio y derrame articular en dos $(9.5 \%)$ de 21 pacientes con MACI; éstos se resolvieron en las cuatro semanas posteriores al procedimiento. ${ }^{21}$ En otro estudio, dos (5\%) de 41 pacientes desarrollaron trombosis venosa profunda temprana después del tratamiento con MACI, mientras que un paciente desarrolló un hematoma postoperatorio, ${ }^{22}$ los pacientes se recuperaron sin secuelas. Por otro lado, en distintos estudios sobre microfracturas no se reportaron complicaciones durante la aplicación del tratamiento. $23,24,25,26,27,28,29,30$

En nuestro estudio se presentaron dos derrames articulares en el grupo MACI que fueron resueltos de manera satisfactoria con artrotomía, sin secuelas; los riesgos potenciales asociados a la técnica y las habilidades quirúrgicas requeridas con la terapia MACI tienen impacto en las complicaciones postquirúrgicas.

Las fortalezas de este estudio de tipo cohorte retrospectiva se relacionan con la inclusión de un número similar en los dos grupos de estudio, así como las características de edad, sexo, síntomas, localización de la lesión y características funcionales. En el estudio que nos ocupa, debido al diseño retrospectivo, no se tomaron biopsias del tejido articular ni evaluación artroscópica, por lo que son limitaciones para demostrar las características histológicas y macroscópicas del nuevo tejido. Asimismo, otra limitación es la duración del seguimiento a corto plazo posterior al procedimiento quirúrgico.

Los resultados del presente estudio demostraron que el uso de MACI disminuye significativamente el dolor y mejora la función en 12 meses después del tratamiento en pacientes sintomáticos con lesiones condrales $\geq 3 \mathrm{~cm}^{2}$; la mejoría fue significativa en comparación con microfracturas; estas evidencias son una aportación al conocimiento para el tratamiento de la patología condral. Por otro lado, es necesario establecer el protocolo estándar de rehabilitación que permita la evaluación uniforme de los resultados con MACI.

Al final, en este estudio se demostró mejoría significativa en el grupo MACI con alivio del dolor, funcionalidad y arcos de movimiento, en comparación con el tratamiento de $\mathrm{MF}$ en lesiones $\geq 3 \mathrm{~cm}^{2}$ del cartílago articular de rodilla después de un año de seguimiento.

\section{Agradecimientos}

Los autores agradecen a la doctora Ana M. Contreras de la Unidad de Investigación y Desarrollo Tecnológico (UNIDET) Medyarthros por su asesoría en la redacción de este artículo. 
Bibliografía

1. Aroen A, Loken S, Heir S, Alvik E, Ekeland A, Granlund OG, Engebretsen L. Articular cartilage lesions in 993 consecutive knee arthroscopies. Am J Sports Med. 2004; 32(1): 211-5.

2. Davies-Tuck ML, Wluka AE, Wang Y, Teichtahl AJ, Jones G, Ding $\mathrm{C}$, et al. The natural history of cartilage defects in people with knee osteoarthritis. Osteoarthritis Cartilage. 2008; 16(3): 337-42.

3. Sellards RA, Nho SJ, Cole BJ. Chondral injuries. Curr Opin Rheumatol. 2002; 14(2): 134-41.

4. Brandt KD, Dieppe P, Radin E. Etiopathogenesis of osteoarthritis. Med Clin North Am. 2009; 93(1): 1-24, xv.

5. Hangody L, Vásárhelyi G, Hangody LR, Sükösd Z, Tibay G, Bartha $\mathrm{L}$, et al. Autologous osteochondral grafting-technique and long-term results. Injury. 2008; 39 (Suppl 1): S32-9.

6. Alford JW, Cole BJ. Cartilage restoration, part 2: techniques, outcomes, and future directions. Am J Sports Med. 2005; 33(3): 443-60.

7. Mithoefer K, Williams RJ 3rd, Warren RF, Potter HG, Spock CR, Jones EC, et al. Chondral resurfacing of articular cartilage defects in the knee with the microfracture technique. Surgical technique. J Bone Joint Surg Am. 2006; 88 Suppl. 1 Pt 2: 294-304.

8. Williams RJ 3rd, Harnly HW. Microfracture: indications, technique, and results. Instr Course Lect. 2007; 56: 419-28.

9. Mithoefer K, Williams RJ 3rd, Warren RF, Potter HG, Spock CR, Jones EC, et al. The microfracture technique for the treatment of articular cartilage lesions in the knee. A prospective cohort study. $J$ Bone Joint Surg Am. 2005; 87: 1911-20.

10. Brittberg M, Lindahl A, Nilsson A, Ohlsson C, Isaksson O, Peterson L. Treatment of deep cartilage defects in the knee with autologous chondrocyte transplantation. $N$ Engl J Med. 1994; 331(14): 889-95.

11. Peterson L, Minas T, Brittberg M, Nilsson A, Sjogren-Jansson E, Lindahl A. Two to 9-year outcome after autologous chondrocyte transplantation of the knee. Clin Orthop Relat Res. 2000; 374: 212-34.

12. Gooding CR, Bartlett W, Bentley G, Skinner JA, Carrington R, Flanagan A. A prospective, randomised study comparing two techniques of autologous chondrocyte implantation for osteochondral defects in the knee: periosteum covered versus type I/III collagen covered. Knee. 2006; 13(3): 203-10.

13. Brittberg M. Cell carriers as the next generation of cell therapy for cartilage repair: a review of the matrix-induced autologous chondrocyte implantation procedure. Am J Sports Med. 2010; 38(6): 1259-71.

14. Gigante A, Bevilacqua C, Ricevuto A, Mattioli-Belmonte M, Greco F. Membrane-seeded autologous chondrocytes: cell viability and characterization at surgery. Knee Surg Sports Traumatol Arthrosc. 2007; 15(1): 88-92.

15. Knutsen G, Engebretsen L, Ludvigsen T, Drogset JO, Grøntvedt T, Solheim E, et al. Autologous chondrocyte implantation compared with microfracture in the knee: a randomized trial. J Bone Joint Surg Am. 2004; 86-A(3): 455-464.

16. Roos EM, Engelhart L, Ranstam J, Anderson AF, Irrgang JJ, Marx RG, et al. ICRS recommendation document: patient-reported outcome instruments for use in patients with articular cartilage defects. Cartilage. 2011; 2(2): 122-36.
17. Saris DB, Vanlauwe J, Victor J, Haspl M, Bohnsack M, Fortems Y, et al. Characterized chondrocyte implantation results in better structural repair when treating symptomatic cartilage defects of the knee in a randomized controlled trial versus microfracture. Am J Sports Med. 2008; 36(2): 235-46.

18. Guillén-García P, Rodríguez-Iñigo E, Aráuz S, Guillén-Vicente M, Guillén-Vicente I y cols. Nuestra experiencia con la técnica de implante de condrocitos autólogos para el tratamiento de lesiones condrales: resultados de 50 pacientes a 2 años de seguimiento. Revista Española de Artroscopía y Cirugía Articular. 2015; 22(3): 120-5.

19. Basad E, Ishaque B, Bachmann G, Sturz H, Steinmeyer J. Matrix induced autologous chondrocyte implantation versus microfracture in the treatment of cartilage defects of the knee: a 2-year randomized study. Knee Surg Sports Traumatol Arthrosc. 2010; 18(4): 519-27.

20. Marlovits S, Aldrian S, Wondrasch B, Zak L, Albrecht C, Welsch G, et al. Clinical and radiological outcomes 5 years after matrix-induced autologous chondrocyte implantation in patients with symptomatic, traumatic chondral defects. Am J Sports Med. 2012; 40(10): 2273-80.

21. Moseley JB Jr, Anderson AF, Browne JE, Mandelbaum BR, Micheli $\mathrm{LJ}, \mathrm{Fu} F$, et al. Long-term durability of autologous chondrocyte implantation: a multicenter, observational study in US patients. Am J Sports Med. 2010; 38(2): 238-46.

22. Ebert JR, Robertson WB, Woodhouse J, Fallon M, Zheng MH, Ackland $\mathrm{T}$, et al. Clinical and magnetic resonance imaging-based outcomes to 5 years after matrix-induced autologous chondrocyte implantation to address articular cartilage defects in the knee. Am J Sports Med. 2011; 39(4): 753-63.

23. Gill TJ. The treatment of articular cartilage defects using microfracture and debridement. Am J Knee Surg. 2000; 13(1): 33-40.

24. Steadman JR, Briggs KK, Rodrigo JJ, Kocher MS, Gill TJ, Rodkey WG. Outcomes of microfracture for traumatic chondral defects of the knee: average 11-year follow-up. Arthroscopy. 2003; 19(5): 477-84.

25. Knutsen G, Drogset JO, Engebretsen L, Grøntvedt T, Isaksen V, Ludvigsen TC, et al. A randomized trial comparing autologous chondrocyte implantation with microfracture: findings at five years. $J$ Bone Joint Surg Am. 2007; 89(10): 2105-12.

26. Kreuz PC, Steinwachs MR, Erggelet C, Krause SJ, Konrad G, Uhl M, et al. Results after microfracture of full-thickness chondral defects in different compartments in the knee. Osteoarthritis Cartilage. 2006; 14 (11): 1119-25.

27. Mithoefer K, McAdams T, Williams RJ, Kreuz PC, Mandelbaum BR. Clinical efficacy of the microfracture technique for articular cartilage repair in the knee: an evidence-based systematic analysis. Am J Sports Med. 2009; 37(10): 2053-63.

28. Mithoefer K, Saris DB, Farr J, Kon E, Zaslav K, Cole BJ, et al. Guidelines for the design and conduct of clinical studies in knee articular cartilage repair: International Cartilage Repair Society recommendations based on current scientific evidence and standards of clinical care. Cartilage. 2011; 2(2): 100-21.

29. Vanlauwe J, Saris DB, Victor J, Almqvist KF, Bellemans J, Luyten FP. Five-year outcome of characterized chondrocyte implantation versus microfracture for symptomatic cartilage defects of the knee: early treatment matters. Am J Sports Med. 2011; 39(12): 2566-74.

30. Ogura T, Mosier B, Bryant T, Minas T. A 20-year follow-up after firstgeneration autologous chondrocyte implantation. Am J Sports Med. 2017; 45(12): 2751-61. 